\title{
The effects of $R f 5$ and $R f 6$ on fertility restoration in Honglian-type cytoplasmic male sterile (CMS) lines of japonica rice (Oryza sativa L. ssp. japonica)
}

Honggen Zhang ( $\square$ zhg@yzu.edu.cn )

Yangzhou University

Ruixuan Wang

Yangzhou University

Zuopeng Xu

Yangzhou University

Xiangqiang Zhao

Nantong University

Hailin Gao

Yangzhou University

Qiaoquan Liu

Yangzhou University

Shuzhu Tang

Yangzhou University

\section{Research Article}

Keywords: japonica rice, HL-type CMS, Restorer-of-fertility gene (Rf), Restoration ability, Marker-assisted selection

Posted Date: March 18th, 2021

DOl: https://doi.org/10.21203/rs.3.rs-319365/v1

License: (c) (1) This work is licensed under a Creative Commons Attribution 4.0 International License. Read Full License 


\section{Abstract}

The Honglian ( $\mathrm{HL}$ )-type cytoplasmic male sterility (CMS) has only been used in the development of threeline indica rice hybrids, and the fertility of HL-type indica CMS lines can be restored by two nonallelic fertility-restorer $(R f)$ genes, $R f 5$ and $R f 6$. For the development of HL-type japonica hybrid combinations, it is therefore necessary to determine whether $R f 5$ and $R f 6$ can restore the fertility of HL-type japonica CMS lines. Here, we genetically characterized HL-type japonica CMS lines and the ability of $R f 5$ and $R f 6$ to restore fertility for breeding HL-type japonica hybrids. $\mathrm{I}_{2}$-KI pollen staining revealed that HL-type japonica CMS lines and their derived testcross $F_{1}$ hybrids had stained abortive pollen grains, unlike HL-type indica CMS lines. Crossing experiments showed that $R f 5$ and $R f 6$ partially restored the fertility of HL-type japonica CMS lines, and $R f 6$ showed higher restorability than $R f 5$. Furthermore, we found that there were additive and dosage effects of $R f 5$ and $R f 6$ with respect to fertility restoration in HL-type japonica CMS lines. These results give critical insight into the breeding of HL-type japonica CMS lines and restorers, which will be helpful for the development of commercial HL-type japonica hybrids.

\section{Introduction}

Rice is the most important staple food crop that feeds more than half of the world's population, and the breeding of high-yielding rice varieties is very important to meet the requirements of an increasing world population. In the past decade, rice yield has increased significantly with the introduction of semi-dwarf varieties and the exploitation of heterosis, and hybrid rice technology is considered to be a major strategy for increasing the yield potential of rice (Cheng et al. 2007). In breeding practice, three-line and two-line systems are the main strategies for development of hybrid rice. The three-line hybrid breeding system is composed of a cytoplasmic male sterile (CMS) line, a maintainer line and a restorer line (Fujimura et al. 1996; Yuan 1994). Currently, three-line indica hybrids are grown on a large scale, but the planting area of japonica hybrid rice has been limited to around 100,000 ha, accounting for $<3 \%$ of the total japonica rice grown in China (Ma and Yuan 2015; Deng et al. 2006). Therefore, the development and application of japonica hybrids will be an important way to increase the total rice grain yield in China.

The development of three-line hybrid rice is based on CMS, and Wild abortive (WA), Honglian (HL), and Chinsurah Boro II (BT) are three representative types of CMS used for commercial hybrid rice seed production. The BT-type CMS is mainly utilized in japonica hybrid cultivation, while the WA-type and HLtype CMS systems are widely used for three-line indica hybrids (Chen and Liu 2014; Huang et al. 2014; Li et al. 2007; Yuan 1994). Seeds resulting from self-pollination in some BT-type CMS lines and the potential genetic vulnerability caused by intensive use of BT-type CMS are considered to be two main constraints in hybrid japonica rice breeding. (Li et al. 2007; Tang et al. 2005; Tang et al. 2008; Wang et al. 1991). In order to overcome the deficiencies of the BT-type CMS lines, the incorporation of WA- and HL-type CMS into japonica hybrids has been proposed as a selection strategy in the past (Zheng et al. 2020). In a previous study, WA-type and HL-type japonica CMS lines were found to have more stable sterility than BT-type japonica CMS lines (Zhu et al. 2010). However, the fertility restoration in WA-type japonica CMS lines is extremely poor, resulting in the lack of restorer lines in japonica, which means that large-scale breeding 
and cultivation of WA-type hybrid japonica rice is not possible. Compared with WA-type japonica CMS lines, fertility in HL-type japonica CMS lines can be restored more easily, which means that it is possible to breed three-line japonica hybrids based on the HL-type CMS (Tang et al. 2005; Zhu et al. 2010). In addition, the $\mathrm{HL}$ - and BT-type CMS lines share similar restoration and maintenance relationships, and the HL-type japonica CMS lines can be bred easily with the BT-type japonica maintainer by backcrossing (Tan et al. 2008; Zhu et al. 2010). In japonica rice varieties, only BT-type restorer lines have been bred and used in the development of hybrids. However, it has been shown that most BT-type japonica restorer lines have only partial fertility restorability to HL-type japonica CMS lines (Zhang et al. 2016; Zhu et al. 2010). Thus, breeding of HL-type japonica restorer lines with strong restorability is the most important and urgent issue in the development of HL-type japonica hybrids.

CMS can be restored by the presence of a fertility restorer gene $(R f)$ in the nucleus, and $R f$ genes in restorer lines play a fundamental role in the spikelet fertility of $F_{1}$ hybrids. Thus, the genetic characterization of $R f$ genes can provide crucial information for breeding restorer lines. Usually, the $\mathrm{CMS} / R f$ systems act in a target-specific manner, meaning that $R f$ genes are highly specific in the fertility restoration of the CMS lines. To date, several $R f$ genes have been identified in rice. $R f 3$ and $R f 4$, two major fertility restorer genes located on chromosomes 1 and 10, respectively, have been identified for the recovery of pollen fertility in WA-CMS lines (Ahmadikhah and Karlov 2006; Tang et al. 2014; Zhang et al. 1997). Rf5 and Rf6, are located on chromosomes 10 and 8, respectively, and have been mapped and cloned for HL-CMS (Hu et al. 2012; Huang et al. 2000; Huang et al. 2012; Huang et al. 2015). The restorer genes Rf1a/ Rf1b on chromosome 10 for BT-CMS (Akagi et al. 1996; Komori et al. 2004; Wang et al. 2006), Rf17 on chromosome 4 for Chinese wild rice (CW)-CMS (Fujii and Toriyama 2009), and Rf2 on chromosome 2 for Lead rice-type CMS (Itabashi et al. 2011), have been mapped and cloned. Although the $R f$ genes for HL-type CMS have been extensively investigated in indica, there are few related empirical studies describing the characterization and mapping of $R f$ genes for HL-CMS in japonica rice varieties (Zhang et al. 2016). Thus, analyzing the restorability of $R f 5$ and $R f 6$ in japonica rice is a reasonable choice for breeding HL-type japonica restorer lines, which will be valuable for breeding high yielding HLtype japonica hybrids.

In our previous study, near-isogenic lines (NILs) for the $R f 5$ and $R f 6$ genes and the polygene pyramid lines (PPLs) combining Rf5 with Rf6 were developed in japonica rice (Zhang et al. 2019). Here, crossing experiments were performed using NILs and PPLs as the male parent and HL-type japonica CMS lines as female parents to evaluate the abilities of $R f 5$ and $R f 6$ to restore male fertility in japonica lines. In addition, the functional model and effects of $R f 5$ and $R f \sigma$ on the fertility restoration in japonica were analyzed. Our discovery will assist in analyzing the differences of fertility restoration in HL-type CMS in indica/ japonica genetic backgrounds and strengthen the breeding of japonica restorer lines for the development of HL-type japonica hybrids.

\section{Materials And Methods}

\section{Plant materials}


The HL-type indica CMS line HL-YuetaiA (HL-YTA) and three pairs of isonuclear alloplasmic japonica CMS lines; HL-LiuqianxinA (HL-LQXA) and BT-LQXA, HL-NipponbareA (HL-NIPA) and BT-NIPA, and HLLingfengA (HL-LFA) and BT-LFA were used in this study. Rf5 and $R f 6$ are two major HL-type $R f$ genes identified from the elite restorer line indica '93-11'(Hu et al. 2012; Huang et al. 2015). From 2015-2018, we performed marker-assisted selection (MAS) to develop the NILs for Rf5 and Rf6 in the HL-YTA background in order to identify the effects of $R f 5$ and $R f 6$ on fertility restoration in HL-type indica CMS lines. The NILs for $R f 5, R f 6$ designed $\mathrm{NIL}^{R f 5}$ and $\mathrm{NIL}^{R f 6}$, and the polygene pyramid lines PPL ${ }^{R f 5+R f 6}$ in the BT-NIPA genetic background were developed in our previous study (Zhang et al. 2019). In 2018, the isonuclear alloplasmic japonica lines were used as females in separate crosses with $\mathrm{NIL}^{R f 5}, \mathrm{NIL}{ }^{R f 6}$, and $\mathrm{PPL}^{R f 5+R f 6}$, resulting in a testcross population consisting of nine pairs of $\mathrm{F}_{1}$ hybrids for evaluating the fertility restoring ability of these two $R f$ genes in japonica rice lines. Subsequently, three $\mathrm{F}_{2}$ populations derived from crosses between HL-NIPA and NIL ${ }^{R f 5}, \mathrm{HL}-\mathrm{NIPA}$ and $\mathrm{NIL}^{R f 6}$, and HL-NIPA and PPL ${ }^{R f 5+R f 6}$ were planted in 2019 to further analyze the effects of the $R f$ genes on fertility restoration in HL-type japonica CMS lines. Twenty plants were grown for each CMS line or each testcross $F_{1}$ line under natural conditions in the field. These plant materials were all planted at the experimental field of Yangzhou University in Yangzhou, Jiangsu Province $\left(32^{\circ} 23^{\prime} 24^{\prime \prime} \mathrm{N}, 119^{\circ} 25^{\prime} 3^{\prime \prime} \mathrm{E}\right)$ and in Lingshui, Hainan Province $\left(18^{\circ} 31^{\prime} 52^{\prime \prime} \mathrm{N}\right.$, $\left.110^{\circ} 10^{\prime} 53^{\prime \prime} \mathrm{E}\right)$.

\section{Fertility examination}

The CMS lines, NILs, and testcross $F_{1}$ lines (five plants each) were evaluated for pollen fertility, bagged spikelet fertility and natural spikelet fertility. The natural spikelet fertility levels of all plants in the three $F_{2}$ populations were quantified. For pollen fertility, mature anthers were harvested, smeared in $1 \%$ iodinepotassium iodide $\left(\mathrm{I}_{2}-\mathrm{KI}\right)$ solution, and observed using a light microscope. The numbers of normal darkblue (stainable), clear (unstainable), and typical aborted pollen grains in each individual were counted (Zhu 1979). For bagged spikelet fertility, two major panicles emerging from the sheath on one plant were bagged before flowering. The natural spikelet fertility level and bagged spikelet fertility level were used as the seed-setting rate by counting the filled and unfilled grains from two opening panicles and the two bagged panicles, harvested 25-30 days after flowering.

\section{DNA extraction and PCR}

Total genomic DNA was isolated from fresh leaves of field-grown plants using a cetyltrimethylammonium bromide (CTAB) method with minor modification (Rogers and Bendich 1985), and the resulting DNA concentrations were adjusted to $100 \mathrm{ng} / \mathrm{mL}$ with TE buffer ( $\mathrm{pH}$ 8.0) for subsequent analyses. Marker loci closely linked to Rf5 and Rf6 were developed as described in previous studies (Huang et al. 2012; Zhang et al. 2017). Simple Sequence Repeat (SSR) markers were identified from the Gramene database (http://www.gramene.org/). Newly-developed specific functional markers for $R f 5$ and $R f 6$ were developed based on the sequences (http://www.ncbi.nlm.nih.gov/) using the BLAST algorithm to search the sequences of 'Nipponbare' $(r f 5, r f 6)$ and '93-11' $(R f 5, R f 6)$. The primers used in this study are 
given in Table S1. The primers were synthesized by Sangon Biotech Co., Ltd. (Shanghai). The molecular marker assays were performed out in $20 \mu$ reaction mixtures containing $1 \times$ PCR buffer, $0.1 \mathrm{mmol} / \mathrm{L}$ of each dNTP, $1.0 \cup$ Taq DNA polymerase, $0.2 \mu \mathrm{mol} / \mathrm{L}$ of each primer, and $20 \mathrm{ng}$ template DNA. The amplification reaction consisted of an initial denaturation cycle of $94^{\circ} \mathrm{C}$ for $4 \mathrm{~min}$, followed by 30 cycles of $94^{\circ} \mathrm{C}$ for $45 \mathrm{~s}, 55^{\circ} \mathrm{C}$ for $45 \mathrm{~s}$, and $72^{\circ} \mathrm{C}$ for $50 \mathrm{~s}$, with a final extension step of $72^{\circ} \mathrm{C}$ for $5 \mathrm{~min}$. The amplification products were separated by electrophoresis on a $3.0 \%(\mathrm{w} / \mathrm{v})$ agarose gel containing ethidium bromide, and visualized with a GEL DOC 1000 system (Bio-Rad Company).

\section{Data analysis}

Analysis of variance (ANOVA) as implemented in SPSS14.0 was used for statistical analysis of the fertility of the materials and populations used in this study.

\section{Results}

\section{HL-type CMS japonica lines and the testcross $F_{1}$ hybrids have stainable pollen grains}

In 2018, pollen grains of the CMS lines, $93-11$, the NILs (NIL ${ }^{R f 5}, \mathrm{NIL}^{\text {Rf6 }}$ and $\mathrm{PPL}^{\text {Rf5+Rf6}}$ ) and the testcross $F_{1}$ plants were analyzed using $I_{2}-K I$ staining. The fertile pollen grains of $93-11$ and the NILs stained dark-blue, and the sterile pollen grains of HL-YTA (indica) were spherical and showed no starch accumulation (Fig. 1a, b). The indica $93-11$ cultivar and the NILs displayed normal spikelet fertility (data not shown). The sterile pollen grains of BT-type and HL-type japonica lines were filled with starch, similar with those of $93-11$, and the pollen grains from the BT-type and HL-type japonica lines were morphologically similar (Fig. 1c-h). All the CMS lines used in this study had bagged spikelet fertility levels of zero, indicating that the pollen grains from these lines are completely sterile. Because BT-type and HLtype CMS are gametophytic CMS systems, $50 \%$ or $75 \%$ of pollen grains in the $\mathrm{F}_{1}$ hybrids (carrying one $R f$ gene or two $R f$ genes) can be theoretically restored. However, the pollen grains of testcross $\mathrm{F}_{1}$ plants derived from BT-type CMS japonica lines and HL-type CMS japonica lines stained dark blue with $\mathrm{I}_{2}-\mathrm{KI}$, and the sterile and fertile pollens were morphologically indistinguishable (Fig. 1j-n). Thus, it is obvious that the effect of HL-type CMS on pollen fate differs in the indica and japonica genetic backgrounds, which is consistent with our previous findings (Zhang et al. 2016). Thus, more detailed in-depth studies are needed to understand the underlying mechanisms behind these differences in CMS in indica vs. japonica rice lines.

\section{Development of NILs for Rf5 and Rf6 in the HL-YTA background}

For analyzing the ability of $R f 5$ and $R f 6$ to restore fertility in HL-type indica CMS lines, NILs for $R f 5$ and $R f 6$ were developed in the HL-YTA background by MAS. In 2015, we first crossed HL-YTA with 93 - 11, a restorer line carrying $R f 5$ and $R f 6$. The resulting $\mathrm{F}_{1}$ plant was backcrossed with HL-YTA to produce a population of $120 \mathrm{BC}_{1} \mathrm{~F}_{1}$ plants, and the genotypes of these plants at the $R f 5$ and $R f 6$ loci were screened using two markers RM407 (locus tightly linked to Rf6) and RM6100 (locus tightly linked to Rf5). Based on 
the screening results, five plants carrying only $R f 5$ or $R f 6$ were selected for successive backcrossing with the recurrent parent $\mathrm{HL}-\mathrm{YTA}$, which generated $10 \mathrm{BC}_{2} \mathrm{~F}_{1}$ and $10 \mathrm{BC}_{3} \mathrm{~F}_{1}$ lines. In the $\mathrm{BC}_{3} \mathrm{~F}_{1}$ population, the MAS results showed that all of the plants had the linked markers, and two plants carrying $R f 5$ or $R f 6$ that were phenotypically similar to the recurrent parent were preferentially selected to cross with HL-YTA, resulting in four $\mathrm{BC}_{4} \mathrm{~F}_{1}$ lines, from which two $\mathrm{BC}_{5} \mathrm{~F}_{1}$ lines, here designed as YTA-Rf5 and YTA-Rf6, were obtained (Fig. 2a). YTA-Rf5 and YTA-Rf6 had similar plant morphology to HL-YTA, and can be considered to be NILs for Rf5 and Rf6 (Fig. 2b).

\section{Rf5 and Rf6 partially restore fertility in HL-type japonica lines}

In 2018, YTA-Rf5 and YTA-Rf6 were used as the controls to analyze the effects of $R f 5$ and $R f 6$ on fertility restoration in HL-type japonica CMS lines. The natural spikelet fertility levels of the plants were used as indicators for evaluating the ability of $R f 5$ and $R f 6$ to restorer fertility in HL-type japonica CMS lines. YTARf5 and YTA-Rf6 plants showed spikelet fertility levels of $~ 80 \%$, and no significant differences were observed between these two types of plants (Fig. 3a). The results revealed that $R f 5$ and $R f 6$ exhibited similar restorability and were effective at restoring fertility to HL-type indica lines, which was in agreement with previous reports (Huang et al. 2012; Huang et al. 2015).

In the testcross population, natural spikelet fertility levels of the testcross $F_{1}$ hybrids between BT-type japonica $\mathrm{CMS}$ lines and $\mathrm{NIL}^{R f 5}, \mathrm{NIL}^{R f 6}$, and $\mathrm{PPL}^{R f 5+R f 6}$ were $>79 \%$, indicating that these two genes are effective at restoring fertility in BT-type CMS japonica lines (Fig. $3 \mathrm{~b}$ ). In contrast, the testcross $\mathrm{F}_{1}$ hybrids between HL-type CMS japonica lines and $\mathrm{NIL}^{R f 5}$ showed natural spikelet fertility levels of $~ 30 \%$ (ranging from $27.92-31.31 \%$ ), and the natural spikelet fertility levels of the testcross $F_{1}$ hybrids between HL-type CMS japonica lines and $\mathrm{NIL}^{R f 6}$ were 50\% (ranging from 47.77-56.77\%), which is significantly lower than that of the corresponding testcross $\mathrm{F}_{1}$ hybrids with BT-type CMS lines (Fig. 3b). These results indicate that $R f 5$ and $R f 6$ restore partial fertility to HL-type japonica CMS lines, and the effect of $R f 6$ is larger than that of $R f 5$ on fertility restoration in HL-type japonica CMS lines. Moreover, the natural spikelet fertility levels of the testcross $\mathrm{F}_{1}$ hybrids between the $\mathrm{HL}$-type CMS japonica lines and PPL ${ }^{R f 5+R f 6}$ were > $60 \%$, which is considerably higher than the crosses with $\mathrm{NIL}^{R f 5}$ and $\mathrm{NIL}^{R f 6}$, implying that $R f 5$ and $R f 6$ exert an additive effect on fertility restoration in HL-type japonica CMS lines (Fig. 3b). In addition, similar results were observed for the bagged spikelet fertility levels of the testcross $F_{1}$ hybrids, although they were significantly reduced compared with the natural spikelet fertility levels (data not shown).

\section{Rf5 and Rf6 display dosage effects on fertility restoration in HL-type japonica CMS lines}

In 2019, there were 40, 40, and 100 plants in the HL-NIPA/NIL ${ }^{R f 5}, \mathrm{HL}-\mathrm{NIPA} / \mathrm{NIL}^{\text {Rf6 }}$ and HL-NIPA/PPL ${ }^{\text {Rf5+Rf6 }}$ $\mathrm{F}_{2}$ populations, respectively. Due to outcrossing, the HL-NIPA plants exhibited very low natural spikelet fertility levels $(<2 \%)$ under natural conditions without isolation. Plants in the three $F_{2}$ populations exhibited natural spikelet fertility levels of $\geq 20.43 \%$ at the same time, indicating that all of these $F_{2}$ plants were fertile. Also, we screened the plants in the three $F_{2}$ populations with two molecular markers, 
Rf1a-M1 (Rf5 functional-marker) and 1870CF (Rf6-functional marker). There were 17 Rf-homozygous individuals and 23 heterozygous individuals, and 25 homozygous individuals and 15 heterozygous individuals in the HL-NIPA/NIL ${ }^{R f 5}$ and $\mathrm{HL}-\mathrm{NIPA} / \mathrm{NIL}^{R f 6} \mathrm{~F}_{2}$ populations, respectively, and the segregation ratios of the homozygous and heterozygous genotypes were $1: 1\left(\chi^{2}=0.34\right.$ and 0.11 in the HL-NIPA/NIL Rf5 and HL-NIPA/NIL ${ }^{R f 6} \mathrm{~F}_{2}$ populations, respectively, which were $\left.<\chi_{0.05}^{2}=3.84\right)$. Moreover, we failed to detect plants with the genotypes rf5rf5, rf6rf6, and rf5rf5rf6rf6 in the HL-NIPA/NIL ${ }^{R f 5}, \mathrm{HL}-\mathrm{NIPA} / \mathrm{NIL}^{R f 6}$, and HLNIPA/PPL ${ }^{R f 5+R f 6} \mathrm{~F}_{2}$ populations, respectively. These results indicate that fertility restoration in $\mathrm{HL}$-type japonica CMS lines by $R f 5$ and $R f 6$ is consistent with a gametophytic restoration model.

In the HL-NIPA/NIL ${ }^{R f 5} \mathrm{~F}_{2}$ population, plants harboring the genotype of $R f 5 r f 5$ had an average natural spikelet fertility level of $35.60 \%$, and plants with the Rf5Rf5 genotype displayed an average natural spikelet fertility level of $58.09 \%$ (Fig. $4 \mathrm{a}$ ). In the HL-NIPA/NIL ${ }^{R f 6} \mathrm{~F}_{2}$ population, the average natural spikelet fertility levels of the plants carrying the Rf6rf6 and Rf6Rf6 genotypes were $47.33 \%$ and $71.79 \%$, respectively (Fig. 4b). In the HL-NIPA/PPL ${ }^{R f 5+R f 6} \mathrm{~F}_{2}$ population, plants carrying the Rf5rf5rf6rf6 genotype had an average natural spikelet fertility of $28.54 \%$, which was the lowest among all of the $F_{2}$ plants. In contrast, plants carrying the Rf5Rf5Rf6Rf6 genotype had the highest natural spikelet fertility of $78.07 \%$ (Fig. 4c). These results indicate that there are dosage effects associated with $R f 5$ and $R f 6$ on fertility restoration in the HL-CMS japonica lines.

\section{Discussion}

Development of three-line hybrid rice depends on the breeding of elite CMS lines and restorers. In China, three-line japonica hybrids have been available since 1976 and are based mainly on the BT-type CMS (Shinjyo 1969). To accelerate the development of three-line japonica hybrids, breeders have attempted to develop three-line japonica hybrids using other CMS systems, such as HL-type and WA-type CMS. However, the genetic characters of the HL-type and WA-type CMS japonica lines and restorers have not been well studied, resulting in the failure to breed HL-type and WA-type CMS japonica hybrids. In the present study, we showed that the HL-type japonica CMS lines have aborted pollen grains that stain dark blue with $\mathrm{I}_{2}-\mathrm{KI}$. Two HL-type $R f$ genes, $R f 5$ and $R f 6$, both partially restored fertility of HL-type japonica CMS lines, and we identified a dosage effect of these two genes in the fertility restoration of HL-type japonica CMS lines.

HL-type CMS is derived from common red-awned wild rice (Oryza rufipogon), and has been successfully used for developing three-line indica hybrids, which have been planted in China and Southeast Asia for many years (Li et al. 2007). In indica-type rice, the spherical aborted pollen grains are considered to be one of the representative characteristics of HL-type CMS lines (Li et al. 2007; Rao 1988; Zhu 1984). In this study, we found that the HL-type japonica CMS lines and their derived testcross $\mathrm{F}_{1}$ hybrids all have bluestaining aborted pollen grains, which is in line with our previous observations (Zhang et al. 2016; Zhu et al. 2010). These results further confirm that it is the HL-type CMS that causes the blue-staining aborted 
pollen grains in the japonica genetic background, which is different from the effect of HL-type CMS in indica rice. It has generally been thought that the morphology of aborted pollen grains is determined by the sterile cytoplasm, not the nuclear genetic backgrounds of the CMS lines. Our results clearly show that this is not the case. Thus, we hypothesize that there is a different mechanism underlying the development of aborted pollen grains caused by the HL-type CMS in indica vs. japonica genetic backgrounds, and that different non-restorer genes might exist in japonica and indica maintainer lines. Further studies are needed to test this hypothesis.

In $O$. sativa ssp. indica, $R f 5$, an $\mathrm{HL}$-type $R f$ gene that is present in many different restorers, has been mapped to the same region of chromosome 10. Rf5 has been cloned, showing that it is the same gene as Rf1a, a fertility restorer gene for BT-type CMS (Hu et al. 2012; Huang et al. 2003; Huang et al. 2000; Liu et al. 2004). $R f 6$, the HL-type $R f$ gene in the indica variety '9311' is located on chromosome 8 (Huang et al. 2012). In a previous study, $R f 5$ and $R f 6$ were shown to restore $50 \%$ pollen fertility and normal spikelet fertility to $F_{1}$ plants from a cross between the indica CMS lines and restorers carrying one of the two genes(Huang et al. 2012). In the present study, we constructed NIL populations for Rf5 and Rf6 in the YTA background, and the natural spikelet fertility levels of the NILs were $\sim 80 \%$. Our observations indicated that $R f 5$ and $R f 6$ are indeed effective for fertility restoration in the HL-type indica CMS lines, which is generally consistent with previously reported results. In contrast, the crossing experiments revealed that $R f 5$ and Rf6 restore only partial fertility to the HL-type japonica CMS lines, indicating that the ability of these two genes to restore fertility in HL-type CMS differs between the indica and japonica genetic backgrounds, which lends some support for the different non-restorer genes in the japonica and indica maintainer lines mentioned above. However, the crossing experiments also showed that the recoverability of HL-type japonica CMS lines was lower than that of HL-type indica CMS lines and BT-type japonica CMS lines. In rice breeding, it is generally thought that the CMS lines with aborted pollen grains that stain blue can be restored more easily than the CMS lines with either spherical aborted or typical aborted pollen grains. Thus, our current observations provide new insights for recognizing the recoverability of CMS lines, which is of value for further breeding and utilization of CMS lines in the future.

The abilities of $R f 5$ and $R f 6$ to restore fertility in HL-type indica CMS lines are similar. In the present study, we initially found that $R f 6$ shows higher restorability than $R f 5$ in HL-type japonica CMS lines, although $R f 5$ and $R f 6$ are both weakly effective in HL-type japonica CMS lines. Rf5 and Rf6 encode proteins that belong to the pentatricopeptide repeat (PPR) family, and these two genes share a high degree of sequence homology (Hu 2012; Huang 2015). For processing the HL-CMS-associated transcript atp6orfH79, RF5 is needed to interact with the RNA-processing factor GRP162, but RF6 works with OsHXK6. Thus, RF6 and RF5 function through distinct mechanisms to restore the fertility of HL-type CMS in indica rice lines. It is reasonable to speculate that $R f 5$ and $R f 6$ play different roles in the fertility restoration of $\mathrm{HL}$-type japonica CMS lines, and this is the most likely reason behind their different effects. Therefore, it will be necessary to test whether Rf6 and Rf5 function through the same mechanism in japonica rice varieties. 
In this study, we observed dosage effects of the $R f$ genes on fertility restoration of HL-type japonica CMS lines in plants carrying different genotypes in the $\mathrm{F}_{2}$ populations; this shows that more $R f$ genes combined in the HL-type japonica $\mathrm{F}_{1}$ hybrids is helpful to increase the seed-setting rate. Usually, the seedsetting rate in three-line hybrids should reach $80 \%$ in rice production. However, our present observations showed that pyramiding the $R f 5$ and $R f 6$ gene is still insufficient to restore a normal level of fertility to HLtype CMS japonica lines. Thus, to breed HL-type japonica restorers, other new major $R f$ genes will be required for pyramiding with $R f 5$ and $R f 6$ to recover full male fertility.

In summary, the results of our study will have important implications for the breeding of japonica hybrids that carry HL-type CMS, and should greatly facilitate the further exploitation of heterosis in rice breeding.

\section{Declarations}

Funding: This study was financially supported by the National Natural Science Foundation (Grant No. 31971913 and 32001517); the Natural Science Foundation of Jiangsu Province (Grant No. BK20200927); and the Government of Jiangsu Province (BE2018357, PAPD, YCSL201904, and PL202004).

Conflicts of interest: The authors declare no conflicts of interest.

Ethics approval and consent to participate: Not applicable

Consent to participate: Not applicable

Consent for publication: Not applicable

\section{Availability of Data and Materials:}

All data supporting the conclusions of this article are provided within the article (and its Additional files).

Author Contributions: $\mathrm{HZ}$ performed the data analysis and drafted the manuscript. RW and ZX performed the phenotypic evaluation and analyzed the data. HG participated in the construction of the NIL populations. QL participated in the design of the study. HZ, ST and XZ designed the study and revised the manuscript. All of the authors have read and approved the final manuscript.

\section{References}

Ahmadikhah A, Karlov GI (2006) Molecular mapping of the fertility-restoration gene $R f 4$ for WAcytoplasmic male sterility in rice. Plant Breed 125:363-367

Akagi H, Yokozeki Y, Inagaki A, Nakamura A, Fujimura T (1996) A codominant DNA marker closely linked to the rice nuclear restorer gene, Rf1, identified with inter-SSR fingerprinting. Genome 39:1205-1209

Chen LT, Liu YG (2014) Male sterility and fertility restoration in crops. Annu Rev Plant Biol 65:579-606 
Cheng SH, Zhuang JY, Fan YY, Du JH, Cao LY (2007) Progress in Research and Development on Hybrid Rice: A Super-domesticate in China. Ann Bot 100(5):959-966

Deng HF, He Q, Shu F, Zhang WH, Yang F, Jing YH, Dong L, Xie H (2006) Status and technical strategy on development of japonica hybrid rice in china. Hybrid Rice 21:1-6

Fujii S, Toriyama K (2009) Suppressed expression of RETROGRADE-REGULATED MALE STERILITY restores pollen fertility in cytoplasmic male sterile rice plants. Proc Natl Acad Sci USA 106:9513-9518

Fujimura T, Akagi H, Oka M, Nakamura A, Sawada R (1996) Establishment of a rice protoplast culture and application of an asymmetric protoplast fusion technique to hybrid rice breeding. Plant Tissue Cult Lett $13: 243-247$

Hu J, Wang K, Huang WC, Liu G, Gao Y, Wang JM, Huang Q, Ji YX, Qin XJ, Wan L, Zhu RS, Li SQ, Yang DC, Zhu YG (2012) The rice pentatricopeptide repeat protein RF5 restores fertility in Hong-Lian cytoplasmic male-sterile lines via a complex with the glycine-rich protein GRP162. Plant Cell 24:109-122

Huang JY, Hu J, Xu X, Li SQ, Yi P, Yang DC, Ren FG, Liu XQ, Zhu YG (2003) Fine mapping of the nuclear fertility restorer gene for HL cytoplasmic male sterility in rice. Bot Bull Acad Sin 44:285-289

Huang JZ, E ZG, Zhang HL, Shu QY (2014) Workable male sterility systems for hybrid rice: Genetics, biochemistry, molecular biology, and utilization. Rice 7:1-14

Huang QY, He YQ, Jing RC, Zhu RS, Zhu YG (2000) Mapping of the nuclear fertility restorer gene for HL cytoplasmic male sterility in rice using microsatellite markers. Chin Sci Bull 45:430-432

Huang WC, Hu J, Yu CC, Huang Q, Wan L, Wang LL, Qin XJ, Ji YX, Zhu RS, Li SQ, Zhu YG (2012) Two nonallelic nuclear genes restore fertility in a gametophytic pattern and enhance abiotic stress tolerance in the hybrid rice plant. Theor Appl Genet 124:799-807

Huang WC, Yu CC, Hu J, Wang LL, Dan ZW, Zhou W, He CL, Zeng YF, Yao GX, Qi JZ, Zhang ZH, Zhu RS, Chen XF, Zhu YG (2015) Pentatricopeptide-repeat family protein RF6 functions with hexokinase 6 to rescue rice cytoplasmic male sterility. Proc Natl Acad Sci USA 112:14984-14989

Itabashi E, Iwata N, Fujii S, Kazama T, Toriyama K (2011) The fertility restorer gene, Rf2, for Lead Ricetype cytoplasmic male sterility of rice encodes a mitochondrial glycine-rich protein. Plant J 65:359-367

Komori T, Ohta S, Murai NY, Kuraya Y, Suzuki S, Hiei Y (2004) Map-based cloning of a fertility restorer gene, $R f-1$, in rice (Oryza sativa L.). Plant J 37:315-325

Li SQ, Yang DC, Zhu YG (2007) Characterization and use of male sterility in hybrid rice breeding. J Integr Plant Biol 49:791-804 
Liu XQ, Xu X, Tan YP, Li SQ, Hu J, Huang JY, Yang DC, Li YS, Zhu YG (2004) Inheritance and molecular mapping of two fertility-restoring loci for Honglian gametophytic cytoplasmic male sterility in rice (Oryza sativa L.). Mol Genet Genomics 271:586-594

Ma GH, Yuan LP (2015) Hybrid rice achievements, development and prospect in China. J Integr Agr 14:197-205

Rao YS (1988) Cytohistology of cytoplasmic male sterile lines in hybrid rice. In: Smith WH, Bostian LR, Cervantes (eds) Hybrid rice. IRRI, Manila, Philippines, 115-128

Rogers SO, Bendich AJ (1985) Extraction of DNA from milligram amounts of fresh, herbarium and mummified plant tissues. Plant Mol Biol 5:69-76

Shinjyo C (1969) Cytoplasmic-genetic male sterility in cultivated rice, Oryza sativa L. Part II. The inheritance of male sterility. Jpn J Genet 44:149-156

Tan YP, Li SQ, Wang L, Liu G, Hu J, Zhu YG (2008) Genetic analysis of fertility-restorer genes in rice. Biol Plantarum 52:469-474

Tang HW, Luo DP, Zhou DG, Zhang QY, Tian DS, Zheng XM, Chen LT, Liu YG (2014) The Rice Restorer Rf4 for Wild-Abortive Cytoplasmic Male Sterility Encodes a Mitochondrial-Localized PPR Protein that Functions in Reduction of WA352 Transcripts. Mol Plant 7:1497-1500

Tang SZ, Sun Y, Zhang HG, Gu Yj, Lu JF, Tian S, Yu B, Gu MH (2005) Comparison on the characteristics of the isonuclear alloplasmic CMS lines in japonica rice. Chin J Rice Sci 19:521-526

Tang SZ, Zhang HG, Liang GH, Yan CJ, Liu QQ, Gu MH (2008) Reasons and countermeasures of slow development on Three-line Japonica hybrid rice. Hybrid Rice 23:1-5

Wang CL, Tang YG, Liu YS, Zhang ZL, Tang SZ, Shi JD (1991) Study on self-fertility of male sterile line Liuqianxin A with BT Cytoplasm in Oryza sativa L. ssp. japonica. Jiangsu J Agr Sci 7:1-7

Wang Z, Zou Y, Li X, Zhang Q, Chen L, Wu H, Su D, Chen Y, Jx, Luo D (2006) Cytoplasmic male sterility of rice with boro II cytoplasm is caused by a cytotoxic peptide and is restored by two related PPR motif genes via distinct modes of mRNA silencing. Plant Cell 18:676-687

Yuan LP (1994) Increasing yield potential in rice by exploitation of heterosis. In: Virmanni SS (ed) Hybrid rice technology. New developments and future prospects. IRRI, Manila, Philippines, 1-6

Zhang H, Zhang L, Si H, Ge Y, Liang G, Gu M, Tang S (2016) Rf5 is able to partially restore fertility to Honglian-type cytoplasmic male sterile japonica rice (Oryza sativa) lines. Mol Breeding 36:102

Zhang H, Cheng X, Zhang L, Liu Q, Gu M, Tang S (2019) Identifying the genes around Rf5 and Rf6 loci for the fertility restoration of WA-type cytoplasmic male sterile japonica rice (Oryza sativa) lines. Euphytica 
Zhang H, Che J, Ge Y, Pei Y, Zhang L, Liu Q, Gu M, Tang S (2017) Ability of Rf5 and Rf6 to Restore Fertility of Chinsurah Boro II-type Cytoplasmic Male Sterile Oryza Sativa (ssp. Japonica) Lines. Rice 10:2

Zhang G, Lu Y, Bharaj TS, Virmani SS, Huang N (1997) Mapping of the Rf-3 nuclear fertility-restoring gene for WA cytoplasmic male sterility in rice using RAPD and RFLP markers. Theor Appl Genet 94:27-33

Zheng W, Ma Z, Zhao M, Xiao M, Sui G (2020) Research and Development Strategies for Hybrid japonica Rice. Rice 13.

Zhu YG (1979) Studies on male sterile lines of rice with different cytoplasms. Acta Agron Sin:29-38

Zhu YG (1984) Study on relationship between restoring and maintaining of different types of male sterile lines in Oryza saliva. Hereditas 6:14-16

Zhu ZB, Zhang HG, Liu C, Li P, Yi CD, Tang SZ, Gu MH (2010) Comparative study on breeding utilization characteristics of the isonuclear alloplasmic japonica CMS lines Liuqianxin A with four different cytoplasm sources. Acta Agron Sin 36:1-8

\section{Tables}

Table 1 Number of plants with different genotypes at the $R f 5$ and $R f 6$ loci in the three $\mathrm{F}_{2}$ populations

\begin{tabular}{|c|c|c|}
\hline Population & Genotype & Number of plants \\
\hline \multirow{2}{*}{ HL-NIPA/NIL ${ }^{R f 5} \mathrm{~F}_{2}$} & Rf5Rf5 & 17 \\
\hline & Rf5rf5 & 23 \\
\hline \multirow[t]{2}{*}{ HL-NIPA/NIL ${ }^{R f 6} \mathrm{~F}_{2}$} & Rf6Rf6 & 25 \\
\hline & Rf6rf6 & 15 \\
\hline \multirow[t]{8}{*}{ HL-NIPA/PPL ${ }^{R f 5+R f 6} \mathrm{~F}_{2}$} & Rf5Rf5Rf6Rf6 & 12 \\
\hline & Rf5Rf5Rf6rf6 & 23 \\
\hline & Rf5rf5Rf6Rf6 & 17 \\
\hline & Rf5rf5Rf6rf6 & 21 \\
\hline & rf5rf5Rf6Rf6 & 10 \\
\hline & rf5rf5Rf6rf6 & 7 \\
\hline & Rf5Rf5rf6rf6 & 4 \\
\hline & $R f 5 r f 5 r f 6 r f 6$ & 6 \\
\hline
\end{tabular}




\section{Figures}

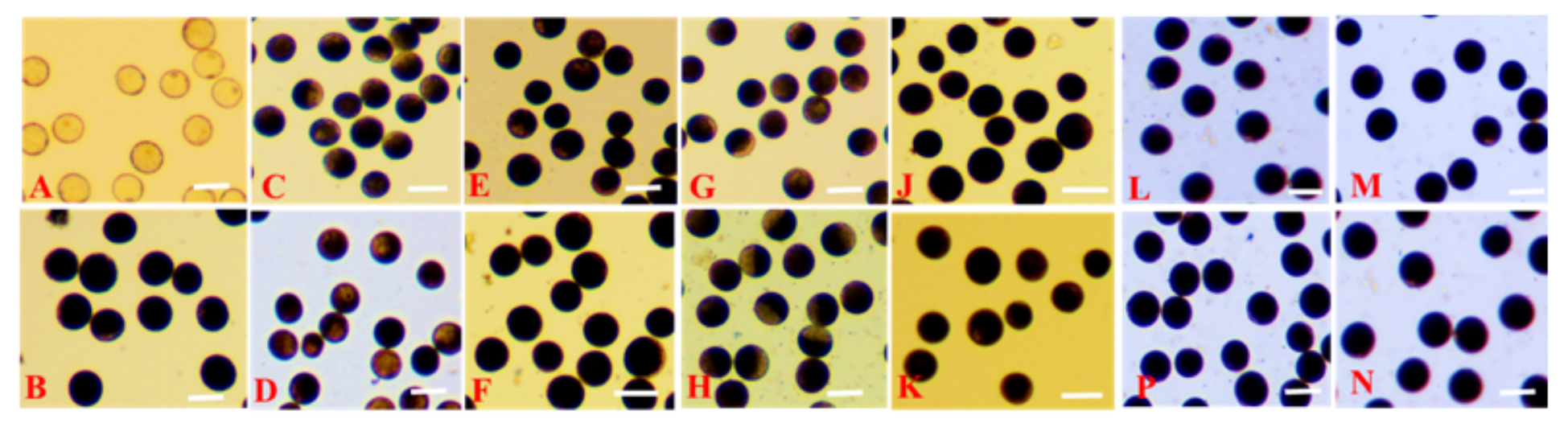

Figure 1

Pollen grains from the parental lines and F1 hybrids stained with I2-KI. (a) HL-YTA (indica). (b) 93-11 (indica). (c-h) Pollen grains from HL-NIPA, BT-NIPA, HL-LqXA, BT-LqXA, HL-LFA, and BT-LFA, respectively. (jn) Pollen grains from the F1 hybrids HL-NIPA/NILRf5, BT-NIPA/ NILRf5, HL-NIPA/NILRf6, HLNIPA/PPLRf5+Rf6, BT-NIPA/ PPLRf5+Rf6, and BT-NIPA/NILRf6, respectively (data from other F1 hybrids were similar but are not shown). Scale bars $=50$ um.
A
Yangzhou, 2015
HL-YTA $\times$ 93-11
Hainan, 2015
HL-YTA $\underset{F_{1}}{ }$
Yangzhou, 2016 HL-YTA BC $_{1} \mathbf{F}_{1}$ (120 plants)

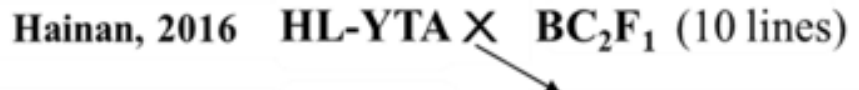
Yangzhou, 2017 HL-YTA $\times \mathbf{B C}_{3} \mathbf{F}_{1}$ (10 lines)
Hainan, 2017 HL-YTA $\underset{\downarrow}{\times} \mathrm{BC}_{4} \mathrm{~F}_{1}$ (4 lines)
Yangzhou, $2018 \quad \mathrm{BC}_{5} \mathrm{~F}_{1}$

(2 lines, YTA-Rf5 and YTA-Rf6)

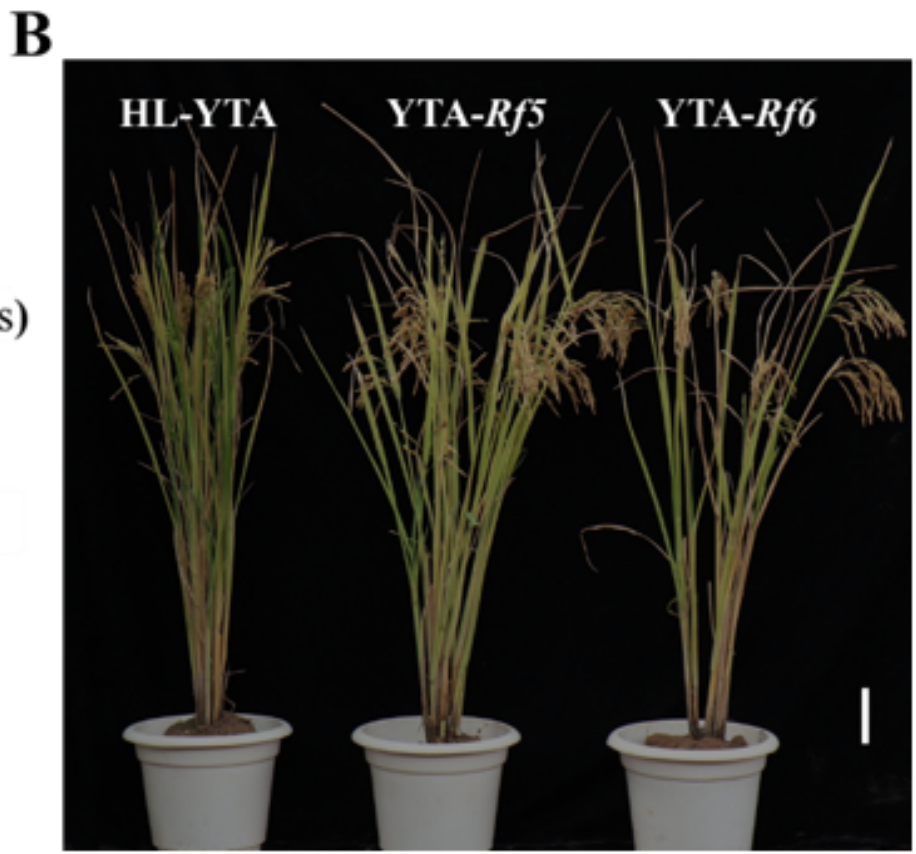

Figure 2

Breeding scheme and gross plant morphology of the parental lines and the newly developed NILs (near isogenic lines) in the YTA genetic background. (a) Breeding scheme showing the development of the YTA- 
Rf5 and YTA-Rf6 NILs. (b) Mature plants of HL-YTA, YTA-Rf5, and YTA-Rf6. Scale bar $=15 \mathrm{~cm}$.

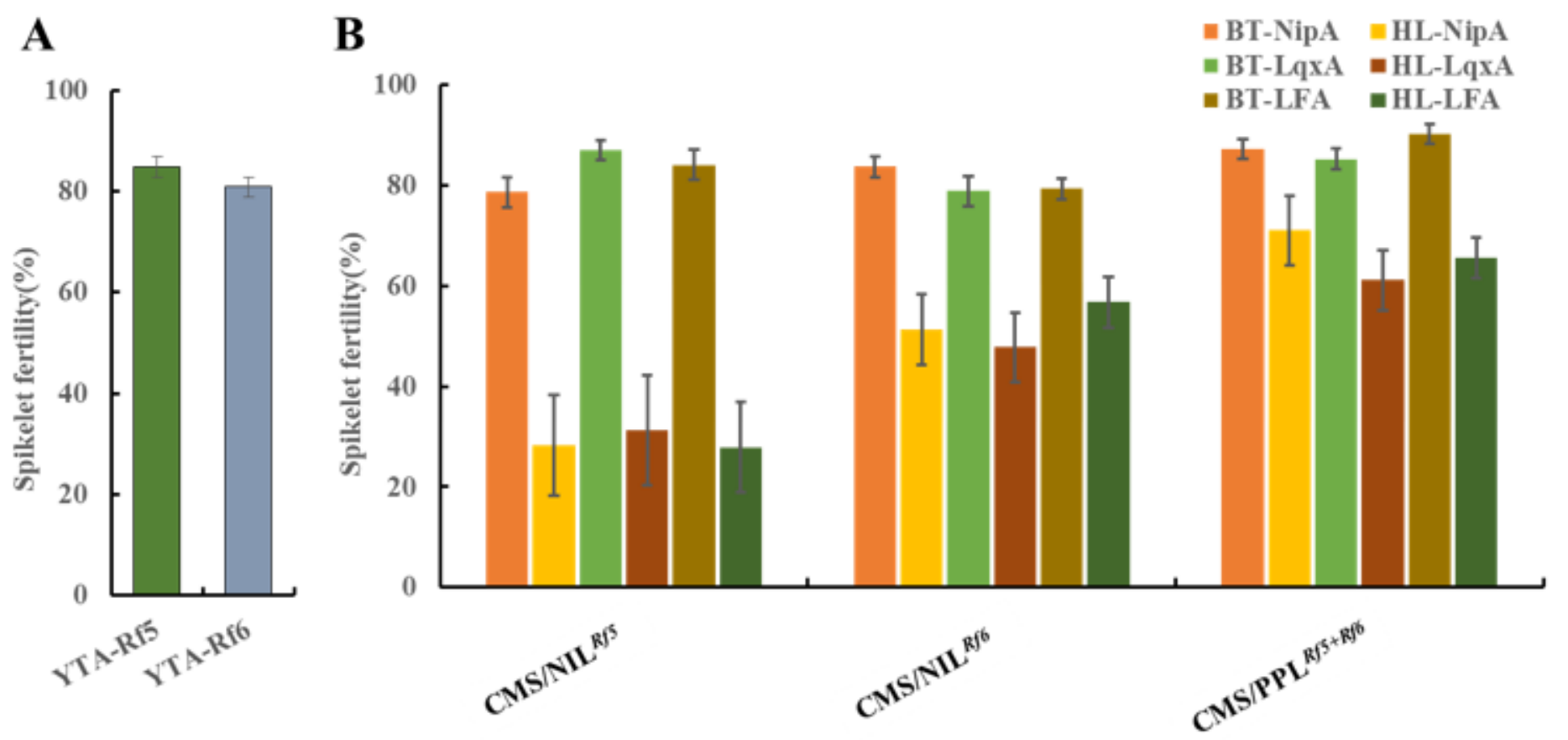

Figure 3

Natural male fertility levels of plants developed in this study. (a) NILs for Rf5 and Rf6 in the YTA background. (b) Testcross F1 plants from crosses between six male-sterile japonica lines, BT-NIPA, BTLQXA, BT-LFA, HL-NIPA, HL-LQXA, and HL-LFA, and the three parental lines CMS/NILRf5, CMS/NILRf6, and CMS/PPLRf5+Rf6.
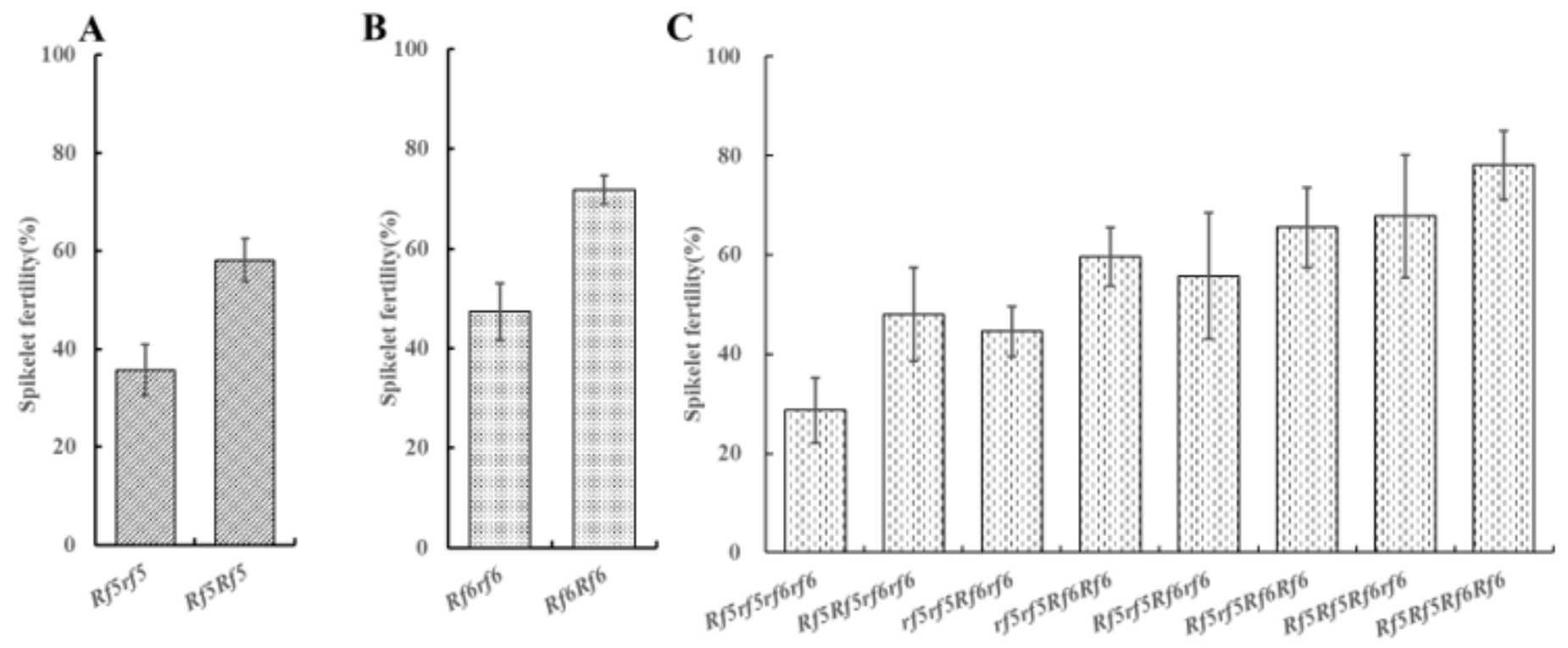

Figure 4 
Natural fertility levels of plants with different Rf genotypes in the F2 populations. (a) The HL-NIPA/NILRf5 F2 population, (b) the HL-NIPA/NILRf6 F2 population, and (c) the HL-NIPA/PPLRf5+Rf6 F2 population. 